\title{
Development of Gating Foils To Inhibit lon Feedback Using FPC Production Techniques
}

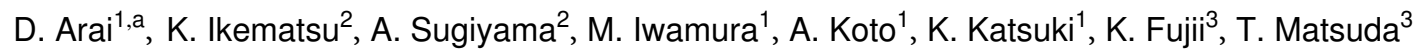 \\ ${ }^{1}$ Fujikura Ltd., 1440 Mutsuzaki, Chiba 285-8550, Japan \\ ${ }^{2}$ Saga U., Saga 840-8502, Japan \\ ${ }^{3}$ High Energy Accelerator Research Organization (KEK), 1-1 Oho, Tsukuba 305-0801, Japan
}

\begin{abstract}
Positive ion feedback from a gas amplification device to the drift region of the Time Projection Chamber for the ILC can deteriorate the position resolution. In order to inhibit the feedback ions, MPGD-based gating foils having good electron transmission have been developed to be used instead of the conventional wire gate. The gating foil needs to control the electric field locally in opening or closing the gate. The gating foil with a GEM (gas electron multiplier)-like structure has larger holes and smaller thickness than standard GEMs for gas amplification. It is known that the foil transmits over $80 \%$ of electrons and blocks ions almost completely. We have developed the gating foils using flexible printed circuit (FPC) production techniques including an improved single-mask process. In this paper, we report on the production technique of $335 \mu \mathrm{m}$ pitch, $12.5 \mu \mathrm{m}$ thick gating foil with $80 \%$ transmittance of electrons in ILC conditions.
\end{abstract}

\section{Introduction}

Time Projection Chamber (TPC) with a Micro Pattern Gas Detector (MPGD) readout has been proposed as a central tracker of International Large Detector (ILD) for the International Linear Collider (ILC) [1]. GEM is one of the MPGD options to be used of the ILC-TPC gasamplification device [2]. F. Sauli proposed the concept of GEM as a gating device [3]. Despite ion-blocking potential of GEM, a small portion of back-flow ions are accumulated during beam collisions. Ion discs are formed in the drift volume of TPC due to a very slow drift velocity of the ions. As the primary electrons pass through the ion discs on the way to the amplification module, the position resolution of TPC might be deteriorated [4]. Using a gating device to stop the ion feedback from the amplification module is effective at preventing this problem thanks to the ILC beam-train structure. This device can be used to stop ions back-flow simply by reversing the electric field in the holes of the gating foil and opening or closing the gate. Achieving a high electron transmission in a large magnetic field for an open GEM gate demands that certain conditions to be fulfilled. Simulation studies showed that only a large aperture (large hole diameter and narrow rim) and very thin foil could meet our requirements [5]. Conventional wire gating was another possibility. However, the device needs a mechanical structure to maintain wire tension, and the electron trajectories may be distorted due to $\mathrm{E}$ x B effects near wire. Nevertheless, the requirement of an optical aperture larger than $80 \%$ is challenging even for FPC manufacturers who specialize in processing

\footnotetext{
a e-mail: daisuke.arai@jp.fujikura.com
}

of fine electrodes. This report describes the requirements for gating foil for ILC-TPC in section 2, R\&D of production methods of the gating foil in section 3, and finally limitations of application of the process in section 4 .

\section{Requirements for gating foil}

As ILC-TPC is operated in a 3.5 T magnetic field, the diffusion of the electrons can be reduced by a gas mixture with a high $\omega \tau$. The ILC-TPC requires a good position resolution of less than $100 \mu \mathrm{m}$, which can be obtained even for a long drift length of up to $2.3 \mathrm{~m}$. The performance of electron transmission through gating foil is also affected by the high magnetic field. It is understood that the rate is closely related to the optical aperture of the gate structure [6]. The prototype gating foil has been studied in a 1 $\mathrm{T}$ magnetic field and shown an electron transmission rate greater than $80 \%$. The results from the study above and the simulation study showed that $80 \%$ transmission was obtained with proper potential setting of the gating foil in a $3.5 \mathrm{~T} \mathrm{~B}$ field. We have extended the area of the gating foil from the $100 \mathrm{~mm} \times 100 \mathrm{~mm}$ for the test module to the $170 \mathrm{~mm} \times 220 \mathrm{~mm}$ for the ILC-TPC module keeping the same specifications as the prototype. The $80 \%$ optical aperture and $12.5 \mu \mathrm{m}$ insulator thickness are needed to satisfy over $80 \%$ electron transmission. The gating foil should have a hole diameter of less than $300 \mu$ m to keep electron position information. The details of requirements for a gating foil and an amplification GEM for ILC-TPC are summarized in Table 1. 
Table 1. Requirement specs of gating foil and amplification GEM for ILC-TPC

\begin{tabular}{|c|c|c|}
\hline Item & Gating foil & Amplification GEM \\
\hline \hline Optical aperture ratio & $\geq 80 \%$ & $22.7 \%$ \\
\hline Hole size & $\leq 300 \mu \mathrm{m}$ & $70 \mu \mathrm{m}$ \\
\hline Hole pitch & $\leq 335 \mu \mathrm{m}$ & $140 \mu \mathrm{m}$ \\
\hline Rim width (Hole pitch - Hole size) & $\leq 35 \mu \mathrm{m}$ & $70 \mu \mathrm{m}$ \\
\hline Insulator thickness & $\leq 12.5 \mu \mathrm{m}$ & $50 \mu \mathrm{m}$ or $100 \mu \mathrm{m}$ \\
\hline Foil size & $170 \mathrm{~mm} \times 220 \mathrm{~mm}$ & $170 \mathrm{~mm} \times 220 \mathrm{~mm}$ \\
\hline \multicolumn{2}{|c}{} \\
\hline
\end{tabular}

\section{R\&D of gating foil production}

Many different processes, including single mask, double mask and laser drilling, have been applied to make amplification GEMs [7]. Each processing method uses photolithography and insulator removing techniques. Those are also used in FPC production processes such as a circuit formation and a though-hole formation process. We have developed prototypes of the gating foil considering suitable conditions for each process and improvements. The following subsections briefly describe the process we have used in developing the gating foil.

\subsection{Laser drilling process}

Both the copper and the polyimide layer can be processed at the same time using Gaussian beam of a UV-YAG laser with a $335 \mathrm{~nm}$ wavelength and about a $30 \mu \mathrm{m}$ beam size . We moved the laser beam along a circle to make $300 \mu \mathrm{m}$ holes (see Fig.1). The gating foil has a hole pitch of 330 $\mu \mathrm{m}$, a polyimide thickness of $25 \mu \mathrm{m}$, and a rim width on the front (back) side of $14 \mu \mathrm{m}(28 \mu \mathrm{m})$ on the $10 \mathrm{~mm} \times 10$ $\mathrm{mm}$ area of the test gate. The optical aperture ratio is 75 $\%$. We verified that the same pattern was also able to be formed on $12.5 \mu \mathrm{m}$ thick polyimide foil. The copper electrodes on the front side are apt to come off the polyimide sheet if the rim is narrower than $10 \mu \mathrm{m}$. The acceptable minimum rim width is therefore about $25 \mu \mathrm{m}$, which allows a maximum optical aperture of $77 \%$ with this circle hole structure. The honeycomb structure with the same rim width and pitch can provide an $85 \%$ optical aperture in principle, however, the laser machine cannot handle different shapes other than circles because it is optimized for FPC production.

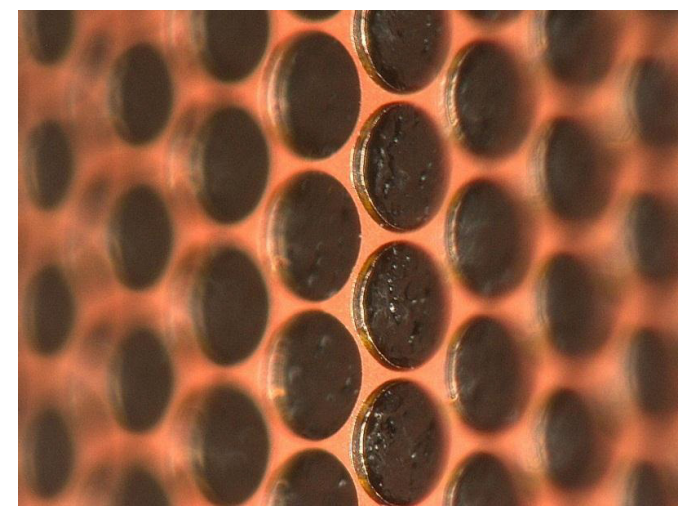

Figure 1. Gating foil produced by laser drilling process. (302 $\mu \mathrm{m}$ hole diameter, $330 \mu \mathrm{m}$ hole pitch and $25 \mu \mathrm{m}$ polyimide thickness on the $10 \mathrm{~mm} \times 10 \mathrm{~mm}$ area)

\subsection{Double mask process}

It is necessary to produce electrode patterns on both sides of gating foil in a double mask process. The insulator is removed by a chemical process or laser etching. In this process, the copper patterns work as etching masks. The accuracy of the photomask alignment on both sides is more than $10 \mu \mathrm{m}$ for large area processing. Creating a $30 \mu \mathrm{m}$ rim with that process is therefore unacceptable. We conclude that the double mask process is not suitable for producing the gating foil.

\subsection{Single mask process}

Although mask alignment in a single mask process is not necessary, how to make an electrode pattern on the opposite side is another issue. We have approached this problem by two methods.

\subsubsection{Single mask process with Ni plating}

We considered using Ni plating to protect the copper electrode formed on the front side when the copper on the back side is etched later. After the circuit production on the front side, a defocused beam of a UV-YAG laser is irradiated to remove only the polyimide insulator where the copper electrode functions as a mask to stop the laser beam. Laser etching enables the creation of less tapered, uniform-quality holes over a wide area than holes created by chemical etching. A UV-YAG laser also seems to cause less damage on the surface of polyimide than a thermal process by a $\mathrm{CO} 2$ laser.

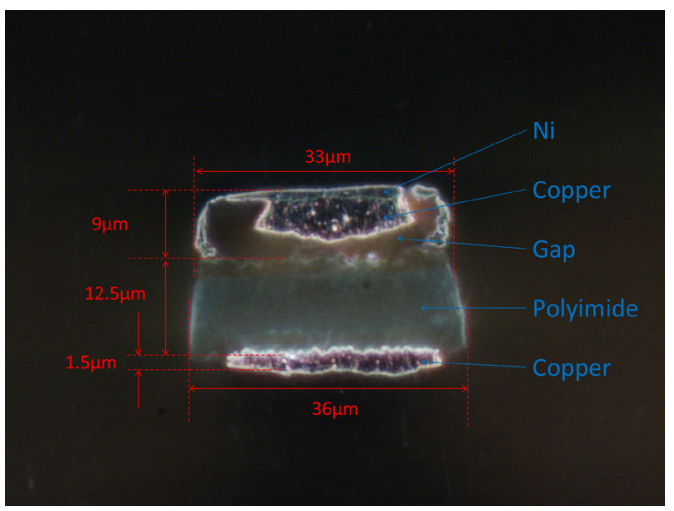

Figure 2. Cross-section view of the gating foil produced by single mask process with $\mathrm{Ni}$ plating

Figure 2 shows the cross-section of the gating foil produced by a single mask process with Ni plating. The gating foil has a polyimide thickness of $12.5 \mu \mathrm{m}$ and a rim width on the back side of $36 \mu \mathrm{m}$. A gap was found between the electrodes ( $\mathrm{Ni}$ and Copper) and the polyimide. The gap was probably caused by the entry of the etching liquid during copper etching on the back side. 


\subsubsection{Single mask process without Ni plating}

We have invented a new single mask process, which allows the copper on the front side to be etched without protecting the copper by $\mathrm{Ni}$. We prepare the sheet having a thicker copper on the front side than the back side so that enough copper is left on the front side when the copper in the hole area of the back side is etched away. After the honeycomb structure circuit on the thick copper side is formed, the polyimide is removed by a defocused beam of a UV-YAG laser. In this process, the thick copper works as a etching mask. Finally, we etched the copper from both sides, where the etching speed in the hole area was two times faster than other areas, and obtained proper patterns of the electrodes on the both sides. Figure 3 shows the schematic view of etching for the back-side copper.

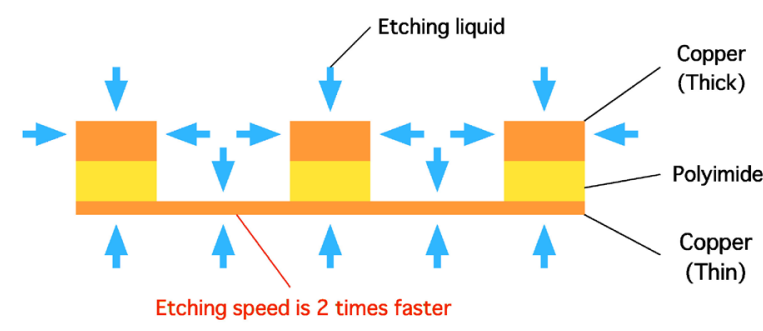

Figure 3. Schematic view of etching of back side copper (The copper of hole area of back sides is etched from both sides, so the etching speed is two times faster.)

Figure 4 and 5 show the gating foil produced by the single mask process without Ni plating. The gating foil has a hole diameter of $304 \mu \mathrm{m}$ on the back side, a hole pitch of $335 \mu \mathrm{m}$, a rim width of $27 \mu \mathrm{m}(31 \mu \mathrm{m})$ on the front (back) side and an insulator thickness of $12.5 \mu \mathrm{m}$ on the $100 \mathrm{~mm} \times 100 \mathrm{~mm}$ area. The optical aperture ratio is $82 \%$. We have produced the gating foil successfully. ILC-TPC Japan group have measured the electron transmission of the gating foil, and verified that the electron transmission was over $80 \%$ under the magnetic field $0 \mathrm{~T}$ and $1 \mathrm{~T}$ [6].

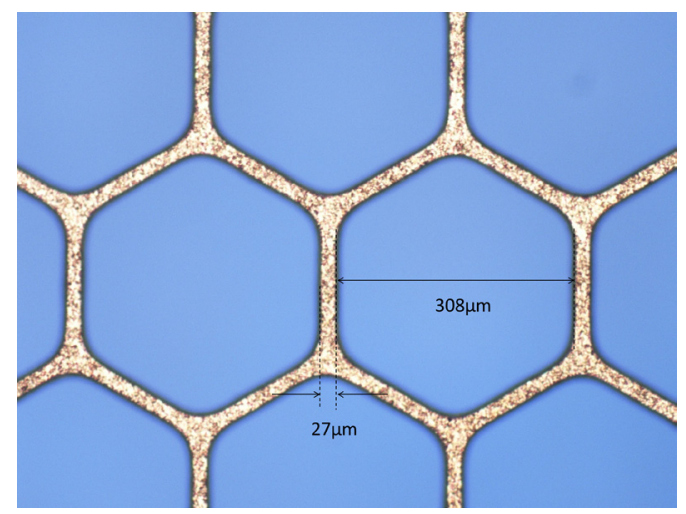

Figure 4. Micrographs of the gating foil (Front side) produced by single mask process without $\mathrm{Ni}$ plating

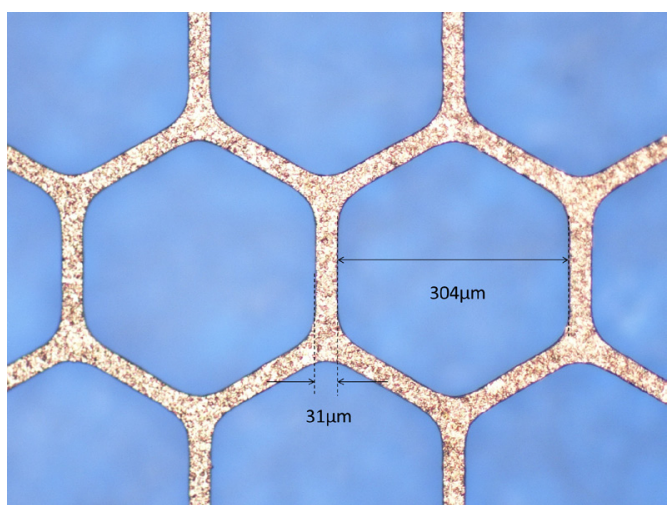

Figure 5. Micrographs of the gating foil (Back side) produced by single mask process without Ni plating

\section{Limitations of gating foil processing}

We have established a stable production process for the gating foil of the $100 \mathrm{~mm} \times 100 \mathrm{~mm}$ test area. However, we still have to know the maximum area for which this method is applicable. In addition, we need to know the minimum limits of the rim width and the hole diameter (keeping the same optical aperture) for further production.

\subsection{Available processing area}

We have to avoid circuit breaks on the front side that lead to electrode breaks in the post etching process. The area to which this process is applicable relies on the probability of a circuit break due to contamination during photolithography. To improve the production environment and optimize the photo-resist, the gating foil with a $30 \mu \mathrm{m}$ electrode width has been successfully produced over the $170 \mathrm{~mm}$ x $220 \mathrm{~mm}$ area of the TPC module.

\subsection{Minimum rim width}

Figure 6 shows the cross-section of the gating foil having a $7 \mu \mathrm{m}(15 \mu \mathrm{m})$ rim width on the front (back) side. The narrow copper of less than $7 \mu \mathrm{m}$ peeled off the polyimide. We conclude the minimum rim width by this process should be $15 \mu \mathrm{m}$.

\subsection{Minimum hole size}

We have tried to make gating foil with a hole diameter of $100 \mu \mathrm{m}$ and a rim width on the back side of $20 \mu \mathrm{m}$ to minimize the hole size. The shape of the electrodes on this foil was not a honeycomb form as shown in Fig.7. The corner of that small hexagonal holes could not be formed using a common chemical process for circuit formation. A diameter of $150 \mu \mathrm{m}$ seems to be the minimum limit of the process. 


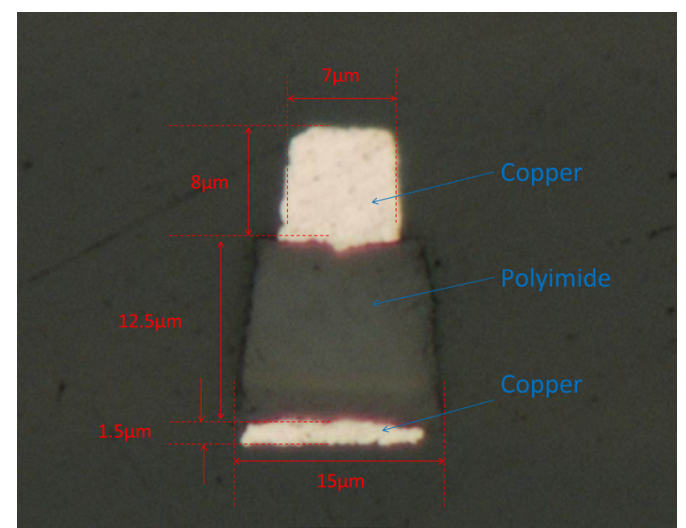

Figure 6. Cross-section view of the gating foil which has a rim width on the front (back) side of $7 \mu \mathrm{m}(15 \mu \mathrm{m})$

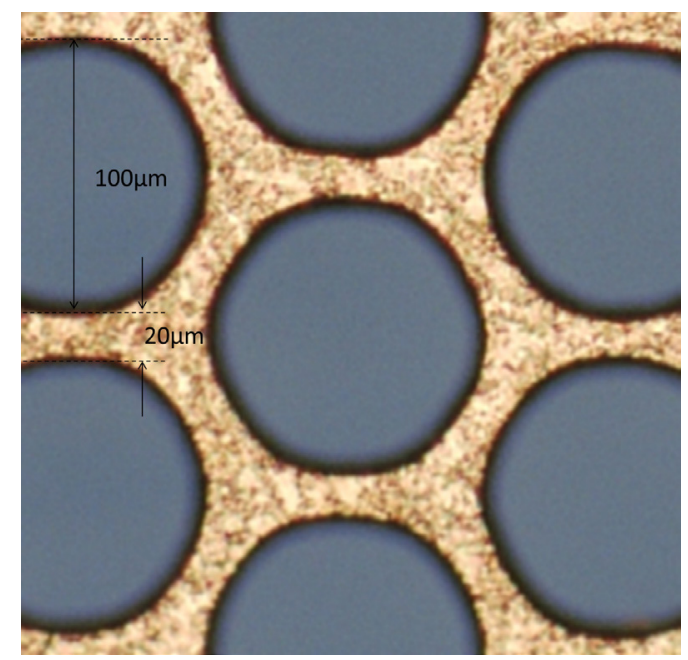

Figure 7. Micrographs of gating foil which has a hole diameter on the front side of $100 \mu \mathrm{m}$

\section{Conclusion}

We have developed the gating foil that has a GEM-like structure and can provide an $82 \%$ optical aperture over the $100 \mathrm{~mm} \times 100 \mathrm{~mm}$ test area and the $170 \mathrm{~mm} \times 230$ $\mathrm{mm}$ module area. This will be a viable gating device to block ion-backflow for ILC-TPC. The gating foil mounted on the ILC-TPC prototype module has been produced and the electron transmission will be measured soon.

\section{References}

[1] The ILC Technical Design Report (TDR) : Volume 4 Detectors, http://www.linearcollider.org/ILC/Publications/ Technical-Design-Report.

[2] R. Yonamine et al. JINST. 7, C06011 (2012)

[3] F. Sauli et al. NIM A560, 269 (2006)

[4] D. Arai, Master thesis (2012), Tokyo University of Agriculture and Technology (TUAT)

[5] P. Gros et al.JINST 8, C11023 (2013)

[6] K. Ikematsu on behalf of the LCTPC-Japan Collaboration, IEEE Nuclear Science Symposium and Medical Imaging Conference (NSS/MIC) 2014 Conference Record (N44-7).

[7] T. Tamagawa et al. NIM A560, 418-424 (2006) 